\title{
Psychological Stress and Antibody Response to Immunization: A Critical Review of the Human Literature
}

\author{
Sheldon Cohen, PhD, Gregory E. Miller, PhD, and Bruce S. Rabin, MD, PhD
}

\begin{abstract}
Objective: The objective of this review was to evaluate the evidence for the hypothesis that psychological stress influences antibody response to immunization in humans. Methods: A critical review of the literature was conducted. Results: The evidence supports an association between psychological stress and suppression of humoral immune (antibody) response to immunization. This association is convincing in the case of secondary immune response but weak for primary response. The lack of consistent evidence for a relation with primary response may be attributed to a failure to consider the critical points when stress needs to be elevated in the course of the production of antibody. Lower secondary antibody responses were found among patients with chronically high levels of stress (severe enduring problems or high levels of trait negative affect). These responses were found most consistently among older adults. Lower secondary responses were also found for those reporting acute stress or negative affect, but only in studies of secretory immunoglobulin A antibody in which psychological and antibody measures were linked very closely in time. Health practices did not mediate relations between stress and antibody responses; however, there were indications that elevated cortisol levels among stressed patients could play a role. Evidence also suggests the possible influences of dispositional stress-reactivity and low positive affect in the inhibition of antibody production. Conclusions: The literature supports a relationship between psychological stress and antibody responses to immunizations. The data are convincing in the case of secondary response but weak for primary response. More attention to the kinetics of stress and antibody response and their interrelations is needed in future research. Key words: stress, antibody, immunization, primary antibody response, psychological stress, secondary antibody response.
\end{abstract}

ELISA = enzyme linked immunosorbent assay; Hep $\mathrm{B}=$ hepatitis $\mathrm{B}$; HI = hemagglutination inhibition; Ig = immunoglobulin; KLH = keyhole limpet hemocyanin; $\mathrm{MHC}=$ major histocompatibility complex; OD = optical density; S-IgA = secretory immunoglobulin A.

\section{INTRODUCTION}

There is substantial evidence that psychological stress alters immune status in humans (see reviews in Refs. 1-4). One criticism of this statement is that it is based primarily on data from in vitro studies. In these studies immune cells or tissues are removed from the body, and their functional capabilities (eg, lymphocyte proliferation and natural killer cell cytotoxicity) are assessed in the laboratory. In vivo studies, in which investigators assess stress-induced changes in naturally occurring immune response (inside the body), are less common. However, the literature addressing the role of stress in in vivo alterations of antibody response, especially responses to immunizations, is rapidly growing. These studies have gained attention not

From the Department of Psychology (S.C., G.E.M.), Carnegie Mellon University; and the Western Psychiatric Institute and Clinic (G.E.M.) and Department of Pathology (B.S.R.), University of Pittsburgh School of Medicine, Pittsburgh, Pennsylvania.

Address reprint requests to: Sheldon Cohen, PhD, Department of Psychology, Carnegie Mellon University, Pittsburgh, PA 15213. Email: scohen@andrew.cmu.edu

Received for publication December 10, 1999; revision received July 10, 2000. only for their potential to establish stress-induced immune changes that occur in vivo but also because of the possible clinical implications of stress altering response to immunization.

In this article we review the human literature on antibody response to several foreign substances (antigens) that stimulate the immune system to produce antibodies. These include immunizations against infectious agents. We provide brief descriptions of the paradigm and of immune processes involved in antibody response, discuss methodological and statistical issues involved in this research, and review the literature organized by the type of antigen. In the case of immunizations against infectious agents, we also address whether the evidence supports clinical differences in protection against disease for those reporting higher levels of stress.

\section{What Is Psychological Stress?}

In this literature psychological stress is usually defined as the experience of negative events or the perceptions of distress and negative affect that are associated with the inability to cope with them (5). Measures vary in terms of how long a person has experienced stress. For example, self-report measures of perceived stress and current affect (last day, week, or month) are thought to assess a relatively short-lived state. Chronic stressors (eg, care giving for an ill spouse or an enduring conflict with a boss) are measures of more longlived experiences. Finally, measures of trait negative affect (average or usual negative emotional tone) are thought to reflect a stable disposition and consequently provide an estimate of negative affective tone 
over very long (years or decades) periods. As discussed later, the duration covered by a measure can be critical to whether a study is sensitive to associations between stress and antibody response.

Although positive affect is not generally considered a measure of stress, we consider it in this article. We do this because reduction in positive affect, like an increase in negative affect, is often a result of confronting negative demands.

\section{What Is Antibody Response?}

The immune system responds to the presence of antigens by producing antibodies (see comprehensive treatments of this topic in Refs. 6 and 7). Antibodies are protein molecules that bind to invading microorganisms and mark them for destruction or prevent them from infecting cells. Antibodies produced in response to a specific antigen will only bind to that antigen. In the studies we review, individuals are injected with an antigen, and the amount of antigenspecific antibody produced is quantified. The more antibody produced in response to an antigen, the more "competent" the humoral immune system is assumed to be. A distinction is made between the first time an antigen is encountered (primary immune response) and subsequent exposures (secondary immune responses). After being sensitized to an antigen by the first exposure, subsequent exposures (secondary responses) are associated with a quicker response, the production of more antibody for a longer duration, and antibody with a higher affinity for the antigen.

Antibody can be measured in serum (IgG and IgM) or in mucosal secretions (S-IgA). IgM antibody is the first class of antibody produced, and it usually can be detected for several weeks to months after antigen exposure. IgG antibodies appear a few days after the IgM antibody but persist for years. S-IgA antibody is produced within a few weeks after antigen exposure and in some case (eg, living antigens) may be continuously produced for years after immunization. IgG and S-IgA classes of antibody provide the long-term protection that is the goal of immunization against infectious agents.

\section{How Is Antibody Produced?}

This section focuses on the immune processes relevant to response to the antigens used in the literature we review. These are mostly protein antigens that are injected, including Hep B surface antigen, influenza (neuraminidase and hemagglutinin) antigens, KLH, and an attenuated rubella virus. The responses to these immunizations are thought to involve relatively simi- lar immunologic processes that we describe below. We also address some differences in response that occur when a protein antigen is orally administered (as in the rabbit albumin studies) and when an injected polysaccharide antigen is used, as in the pneumococcal vaccine study. Readers seeking more advanced treatments of the processes involved in antibody production should consult texts by Benjamini et al. (6) and Rabin (7).

Primary antibody response. The primary antibody response is the response that occurs the first time the immune system encounters an antigen. When antigen is injected into tissue, two pathways are followed simultaneously: (1) Some antigen is taken into antigen presenting dendritic cells that are located in the tissue. The antigen is digested and placed into MHC molecules that carry fragments of the antigen from the cytoplasm of the dendritic cell to the cell membrane. The dendritic cell then moves though lymph vessels to a lymph node. (2) Some of the antigen that remains free moves with lymph fluid through a lymphatic vessel to a lymph node. In the lymph node the free antigen binds to a B lymphocyte that has a specific Ig receptor for the antigen. In contrast, the antigen that is in the MHC molecule on the surface of the dendritic cell binds to another antigen-specific lymphocyte called a T-helper or "CD4" cell. The CD4 cell will then differentiate into a "CD4 TH1" or "CD4 TH2 cell." The differentiated CD4 cell then binds to the antigenspecific B lymphocyte and through a combination of direct activation and release of chemical messengers (called cytokines) activates the B cell to produce antibody to the antigen. The class of antibody that is produced is determined by whether a TH1 or TH2 CD4 cell interacts with the antigen-specific B cell.

Unlike the other injected protein antigens, polysaccharide antigens (like those used in the pneumococcal immunization) are not taken up by dendritic cells. Instead polysaccharides bind directly to specific antibody molecules for the antigen on the membrane of $\mathrm{B}$ cells. Unique characteristics of polysaccharide antigens activate the $\mathrm{B}$ cell to release antibody.

Finally, primary response to the oral administration of antigen varies somewhat from what is described above (8-10). Oral antigens, such as albumin, activate the production of the IgA class of antibody in mucosal secretions. This antibody is termed secretory IgA (S-IgA). S-IgA is produced by plasma cells (mature B lymphocytes) after stimulation by the TH2 class of CD4 cells. This occurs below the surface of the tissue. For example, S-IgA in saliva is produced by plasma cells in the salivary glands and gingiva. Although orally administered antigens can induce antibody production as we have described, oral administration can 
also result in tolerance, the lack of antibody production. Conditions that bias the immune response toward tolerance or immunity are not well understood but may involve the concentration, frequency of exposure, and size of the antigen (11).

Secondary antibody response. The initial response to an antigen activates antigen-specific $\mathrm{T}$ and $\mathrm{B}$ lymphocytes. These cells, now called memory lymphocytes, may persist for many years. When reexposed to the antigen that initially led to their activation, they function more rapidly than during their initial activation. There are several reasons for this. First, there are more antigen-reactive lymphocytes after initial exposure than before exposure. Second, the affinity (tightness) with which B lymphocytes bind to antigen is increased, resulting in more rapid signal transmission for antibody release. Finally, the previously activated lymphocytes express new proteins on their membranes, which promotes more efficient interaction between the $\mathrm{T}$ and $\mathrm{B}$ lymphocytes. Together these changes result in a rapid and increased production of antibody.

Unlike the other antigens discussed in this article, there is no secondary response to pneumococcal antigen. Because the primary response to polysaccharide antigens do not involve T cells (which activate B memory cells), when the antigen is injected a second time, the antibody response is the same as the first time antigen was encountered.

How Can Stress Influence the Production of Antibody?

Psychological stress may alter antibody production through either behavioral or neurobiological pathways (see Refs. 4, 7, 12, and 13 for overviews). For example, stress might alter antibody production through its effects on health practices known to modulate immunity, such as smoking, alcohol consumption, sleep, and diet $(1,14)$. Stress also influences the plasma and tissue concentration of many hormones that bind to specific receptors on the membrane or in the cytoplasm of cells of the immune system, including the various cells that participate in the production of antibodies (see review in Ref. 7). It is likely that the stress-associated hormones having the principal effect on altering antibody production are the glucocorticoids (including cortisol) and catecholamines (including epinephrine and norepinephrine) (7).

Each of the cell types involved in primary and secondary response has receptors for glucocorticoids and catecholamines, and binding of these hormones to their specific receptors alters immune function. Given current knowledge, there is no strong reason to expect that either primary or secondary response would be more susceptible to stress-induced effects. However, it is known that immunosuppressive medications have a more profound effect on the primary than secondary response to antigen (15). Whether this will apply to the effect of stress on immune function remains to be determined.

\section{METHODOLOGICAL ISSUES}

Cross-Sectional, Prospective, and Experimental Designs. All but one study in this literature is correlational. By correlational we mean that these studies are investigating naturally occurring stress conditions (eg, caregivers vs. controls or those with low vs. high levels of a stress as assessed by a self-report instrument). A defining characteristic of these studies is that people are not randomly assigned to more or less stressful conditions (16). Consequently, the possibility exists that third factors (eg, environmental conditions or personal dispositions) exist that select people to high stressed conditions and also cause the suppression of immune response. For example, older people may experience more stress and may also produce less antibody. Obvious third-factor explanations such as age, gender, and race can be eliminated if they are measured. Stressed and nonstressed control groups can be matched on these variables, or these variables can be controlled for (covaried) in statistical analysis. However, even in the best studies, less obvious (and unmeasured) third factors result in some ambiguity in causal interpretation of associations between stress and immune response.

Correlational studies in this literature also vary in regard to whether they use cross-sectional, longitudinal, or longitudinal-prospective designs or analyses. In cross-sectional studies the psychological assessments and antibody assessments are done at approximately the same time; hence the data do not provide sufficient information to determine the direction of causality. Longitudinal studies involve collecting data at more than one point in time. However, a prospective design (and analysis) is required to clarify the direction of causation. In true prospective studies, psychological assessments are done before antigen challenge, and the outcome variable is the change in antibody from before (time of psychological measurement) to after challenge. Because the psychological variables are assessed before exposure to the antigen, this design eliminates the possibility that the response to the antigen caused a psychological change.

Finally, a single study in this literature uses an experimental design in which subjects are randomly assigned to a stress-reducing intervention or placebo 
control group. Experimental designs allow one to make causal inferences (eg, reduced stress resulted in the production of more antibody) without concern about reverse causation or third-factor explanations.

Duration of the Stressor and Timing of Antibody Assessments. If we assume that stress levels need to be elevated at some (unknown) specific points during the primary or secondary production of antibody, then stress would need to be assessed at those appropriate points (see Ref. 17). The need to time stress measures appropriately is most important when assessing "acute" stressful events or affective responses. This is because the short-term fluctuation in stress assessed by this type of measure might occur at a point in the immune response to the antigen where stress does not play a role. Clearly the use of such a measure requires a theory of when stress is most important in this process. However, timing is not an issue when studying chronic or ongoing stressful events or "traitlike" stress characteristics. This is because those with enduring exposure or greater levels of "trait" stress would have elevated stress levels over the entire course of response to the antigen.

What Constitutes a Change in Antibody Level? Antibody measurement is expressed in one of two ways in this literature, as titers or ODs. Studies that assessed $\mathrm{HI}$ express antibody concentrations as a titer. The HI assay capitalizes on the fact that viral-specific antibody binds to a protein that lies on the surface of influenza (and some other) viruses. This protein will usually cause red blood cells to clump together. However, the binding of the viral-specific antibody to the protein inhibits the ability of the viral protein to cause clumping of the red blood cells. Hence clumping provides an indirect measure of the amount of viral specific-antibody bound to the protein. A titer is the reciprocal of the highest dilution of serum having a positive response in the antibody assay. For HI a positive response is when red blood cells do not clump (and hence the antibody is assumed to be present). The sequential testing of different dilutions is generally increased geometrically (eg, 1:2, 1:4; 1:8, etc.). In general, those assessing titers used a traditional (but arbitrary) criterion of a four-fold increase (eg, 1:2 to 1:8) to decide whether an individual has a "significant" increase in viral-specific antibody. For those who start with no detectable antibody, detecting antibody at any concentration (even 1:2) is considered sufficient criterion for a significant change.

Studies that used an ELISA express the antibody level in units of OD. In the ELISA assay, the patient's serum is added to a plastic well coated with the specific antigen of interest. The antibodies in the patient's serum that are specific to that antigen bind to the antigen on the well. Nonbinding antibodies are simply washed away by rinsing the plastic wells, leaving only the antigen-specific antibodies. To quantify the amount of antigen-specific antibody, a second antibody that selectively binds to the antigen-specific antibodies (of a specific class, eg, IgG) is added to the well. An enzyme that is linked to the second antibody causes a color reaction when it binds to the antigenspecific antibody. The intensity of the color is proportional to the concentration of antibody the patient has to the antigen. OD is the measure of the intensity of color and consequently an indirect measure antigenspecific antibody concentration. OD is often reported as an index calculated by dividing the OD of the antigen-coated well by a measure of nonspecific binding to the well (OD of a well not containing antigen). Those assessing OD use more traditional statistical techniques. ELISA is preferable because it is more sensitive (ie, it can detect lower concentrations of antibody) than other serological assays and is evaluated on a continual gradient.

Several of the studies addressing primary antibody response use "seroconversion" as the outcome variable. Seroconversion refers to being able to detect antibody in patients who had no detectable antibody before being immunized. Because the ELISA assay can detect lower concentration of antibody than the HI technique, the ELISA assay may show seroconversion when the HI would not.

Assessments at Baseline. A major methodological concern in these studies is whether baseline antibody levels (before the challenge immunization) are assessed. There are two reasons why this is important. First, baseline assessments help the investigator to distinguish between subjects receiving a primary (no detectable antibody at baseline) or secondary challenge. Second, it is possible that stress is associated with basal levels of antibody. For example, stressed people may not get their annual flu shots. Consequently, when they enter a study, they may start with lower levels of antibody to viruses common to both past inoculations and the challenge immunization in the study. If this is the case, differences between groups after the inoculation (not controlling for baseline) may not be attributable to differential response to the challenge immunization.

\section{RELEVANT RESEARCH}

The review is organized by antigen. We refer readers to the following web sites for information on some of the viral immunization protocols used in these studies: www.cdc.gov/ncidod/diseases/hepatitis/b/factvax and www.cdc.gov/ncidod/diseases/flu/fluvirus.htm. 
Hep $B$. The inoculation that immunizes against the Hep B virus contains a recombinant antigen from the surface of the virus. Antibody that is produced in response to this antigen prevents the virus from infecting liver cells. The immunizations are given as a series of three shots. The first and second inoculations are usually given 1 month apart, and the third is given 6 months after the first. The inoculations are given in series to optimize the antibody response and hence the number of people developing protection against hepatitis (18).

Two studies examined the association between selfreported stress and primary response to Hep B antigen. Both included antibody assessments before the first immunization and approximately 1 month after immunization. Petrie et al. (19) administered a "disclosure" intervention intended to reduce stress (no direct verification of stress reduction) to 20 medical students just before their first immunization. Twenty other students were nondisclosure control subjects. Most of the subjects $(87.5 \%)$ had no antibody at baseline. Petrie et al. failed to find any relation between the intervention and antibody levels at the 1-month follow-up examination. Glaser et al. (20) administered each of the three Hep B immunizations to 48 medical students on the last day of three consecutive examination periods (at first immunization and 1 month and 6 months later). None of the subjects in this study had detectable antibody at baseline. Stress (anxiety and perceived stress) in this study was defined as the cumulative response to all three examination periods, even though two of these measurements occurred after the primary immunization. Seroconversion was less likely to occur after the first immunization in those with higher levels of stress (averaged across the three examination periods) than in those with lower levels of stress. However, there was no association between seroconversion and stress defined solely in terms of the stress level at the time of the first examination (ie, the examination given contiguously with the first inoculation; see Figure 1 in Ref. 20). Subjects who failed to seroconvert in response to the first immunization did, however, report more stress at the second immunization. It is thus possible that the relation between the cumulative stress measure and seroconversion was attributable to the rise in stress that occurred in relation to the second examination (not the first). This explanation assumes that seroconversion could occur as late as several weeks after immunization. Thus, these two studies do not provide evidence that levels of stress at the time of immunization influence primary antibody response to Hep B surface antigen. There is, however, a suggestion that stress occurring weeks after immunization may play a role.
Six Hep B studies (including the two discussed earlier) examined the association of stress and secondary response. Two report cross-sectional analyses examining the relation between stress and antibody levels at a single follow-up. In the Glaser et al. (20) study, stress (anxiety) during the examination period and response to the second immunization were all measured at the time of the third immunization. (Actually, response to the immunization was assessed by a composite variable including antibody titers and an indicator of cellular immune response, blastogenic response to Hep B surface antigen.) They found no association between stress and immune response to the immunization.

In a study of 84 graduate students, Marsland et al. (21) assessed both stress and antibody levels 3 months after the second immunization. They found that trait negative affect (also called neuroticism or emotional instability) was associated with lower levels of antibody. However, state measures of stress (life events and perceived stress) assessed at the same time were not associated with response. Marsland also found that persons who (in later testing) responded to a psychological laboratory stress challenge with greater suppression of an in vitro measure of cellular immune response (phytohemagglutinin-stimulated lymphocyte proliferation) had lower levels of antibody than those who responded with less suppression of the phytohemagglutinin response.

Three studies predict secondary antibody response from stress measured at or just after the prior immunization. In a study of 95 medical students, Jabaaij et al. (22) found that more hassles (minor stressful events) and negative affect at the second and third Hep B immunizations were associated with less antibody production 1 month after the third inoculation. In contrast, Petry et al. (23) studied 81 medical students and found that higher scores on several indices of stress (life events, irascibility, depression, and anxiety) administered on the day of the third immunization were associated with greater production of antibody as assessed 3 months later. Finally, Jabaaij et al. (24) reported a true prospective study of 68 undergraduates, in which they assessed stress as well as antibody 1 month after the second immunization and at the third immunization. A final antibody measure was collected 1 month after the third immunization. They examined subsequent changes in antibody level as a function of stress levels at each assessment. They found no association between hassles and state negative affect assessed at any point and subsequent changes in antibody response. In sum, the results of the correlational studies of secondary Hep B response are inconsistent and inconclusive. 
Finally, Petrie et al. (19) used an experimental design in which 40 medical students were randomly assigned to write an essay about either a highly traumatic personal event or a trivial topic just before the first immunization. The intervention was intended to decrease stress levels in the participants. Unfortunately, the investigators did not assess whether writing about a traumatic event had a lasting effect on subjects' experiences of stress, although some earlier studies have found such effects (eg, Ref. 25). Those who wrote about traumatic events had a greater increase in antibody production than the group who wrote about trivial topics both 3 months after the second immunization and 2 months after the third. Unlike the correlational studies, this unique work provides direct evidence for the argument that stress influences secondary responses. However, replication of this work that included evidence that the intervention had longterm (months) effects on self-reported stress and affect would considerably strengthen our confidence in our interpretation of the results.

$K L H$. KLH is a protein responsible for carrying oxygen in the blood of a marine snail. It is nonpathogenic, and people are not exposed to it naturally. Hence it can be used to assess primary antibody response.

A single study used KLH as an antigen. Snyder et al. (26) inoculated 89 undergraduate women and assessed their KLH-specific antibody (IgG) levels on the day of inoculation and 3 and 8 weeks later. Measures of stress and affect were also collected at the time of immunization and at the 8-week follow-up examination. The measures included stressful life events, daily hassles, and negative and positive affect. None of the traditional stress measures were associated with changes in antibody response from baseline to the 3- or 8-week follow-ups. Nor were there any cross-sectional correlations between stress and antibody concentrations at 8 weeks. Reporting less positive affect on the day of inoculation, however, was associated with producing less antibody by the 8-week follow-up examination.

Influenza Vaccine. There are three studies of antibody response to trivalent (for three viruses) influenza immunizations. Subjects in all three were older adults (age, 53-89 years; medians, $>68$ years). For the most part the influenza studies do not provide enough evidence to know for sure (baseline antibody responses are not always reported) whether they assessed primary or secondary responses. However, in this age group it is reasonable to assume that most, if not all, subjects are seropositive. This is because of the high probability that they have been naturally exposed to the viruses contained in the vaccine or because they had been immunized with the same viral antigens in earlier years. Hence it is assumed that associations in this literature are attributable to differences in secondary response.

Kiecolt-Glaser et al. (27) compared 32 elderly caregivers of spouses with a progressive dementia (hence the caregivers were under chronic stress) with 32 control subjects matched for sex, age, and socioeconomic status. As expected, caregivers reported higher levels of depressive symptoms than control subjects. The investigators assessed antibody on the day of immunization and 1 month and 11/2 months later. Fewer caregivers had a four-fold increase in antibodies (by both HI titers and ELISA) during follow-up than matched control subjects. The reported data did not distinguish between response to the three viruses, and outcome was defined as a four-fold increase in antibodies to any of the three. Consequently the results may be attributable to changes in any (or all) of the vaccine components.

In a subsequent article, Glaser et al. (28) reported two replications of the differences between caregivers and control subjects with an additional group, former caregivers whose spouses had died within the last few years. In the first flu season they studied, they found that both the 23 current and 26 former caregivers produced less antibody in response to the immunization than the 75 control subjects. In a subsequent influenza season, they similarly found that both 32 current and 22 former caregivers were less likely to have a fourfold increase in antibody than the 68 control subjects. Analysis of data in the replications also collapsed across components of the vaccines.

Finally, Vedhara et al. (29) similarly compared 50 elderly caregivers of a spouse with dementia with 67 control subjects who had partners without dementia and were not caregivers. Antibody was assessed the day of immunization and 1, 2, and 4 weeks after immunization. Antibody concentrations to the three vaccine components did not differ between caregivers and control subjects at baseline, and (at least on the average) both groups had detectable antibody to all three viruses. On the day of immunization, caregivers reported more emotional distress (a composite of anxiety, depression, and perceived stress) than control subjects. Over the course of the study, caregivers were less likely than control subjects to show a four-fold increase in antibody in response to any (at least one) of the three vaccine components. Further analyses of the antibody response to each of the viral antigens were done. These analyses included only the 85 subjects who produced some antibody in response to the immunization. Caregivers again produced more antibody than control subjects, but only for the viral antigen (Nanchang A) that was not administered in the previ- 
ous year's vaccine. This result might be explained by the fact that patients who had been immunized in the previous year would begin with high levels of antibody to viral antigens used in the previous year's vaccines. Consequently they would have less variable response to those viral antigens than to new ones.

The results of the influenza vaccine studies are quite provocative. Overall they suggest that elderly people confronted with an enduring and severe stressor develop antibody to influenza immunizations at a slower rate (and possibly less antibody overall) than nonstressed control subjects. Because the probability of becoming infected by an influenza virus generally decreases with increased serum antibody levels (3032 ), these data suggest that stress at immunization is associated with decreased protection (up to 6 weeks after immunization). Longer follow-up periods, however, would help clarify whether the association between stress and antibody level is attributable to a sustained reduction in protection or with just a slowing in the rate of antibody production.

Rubella Vaccine. Only one study has used a liveattenuated virus for immunization. In this study 240 12-year-old girls were administered a set of psychological measures before immunization with the rubella vaccine (33). Antibody levels (assessed by ELISA) to rubella were assessed at baseline and 101/2 weeks later. The psychological assessments included four measures that can be viewed as assessing trait negative affect; neuroticism (tendency to experience negative affect), internalization (trait anxiety, depression, and withdrawal), externalization (trait aggressiveness and destructiveness), and self-esteem. As discussed earlier, trait negative affect assesses differences in affective tone that would be stable over the entire course of this study. Among the girls who were seronegative at baseline (for whom this was a primary antibody response), greater internalization and neuroticism and less selfesteem were all associated with smaller antibody response to the rubella virus. Among the seropositive group (for whom this was a secondary antibody response), there were no associations between psychological measurements and antibody response 101/2 weeks after immunization.

Rabbit Albumin Administered Orally. Stone et al. have reported two studies on the roles of stress and affect in S-IgA antibody production in response to an ingested antigen. In the first study (34), 30 dental students ingested a pill containing rabbit albumin on a daily basis for 10 consecutive weeks. Rabbit albumin is a nonpathogenic protein that is responded to by the immune system with the production of antibody. On Mondays, Wednesdays, and Fridays of the last 8 weeks of the study, subjects completed a daily diary that included positive and negative mood checklists. They also collected saliva samples on these same days for analysis for S-IgA antibody to rabbit albumin. To control for differences in salivary flow, Stone et al. calculated the percentage of all of the S-IgA measured that was rabbit albumin-specific S-IgA (specific S-IgA/total S-IgA). Subjects had lower antigen-specific S-IgA levels on days that they reported more negative moods than usual and on days that they reported less positive moods than usual. Further analyses indicated that positive and negative moods were making equivalent contributions to the relation. However, these effects are cross-sectional, and the authors did not report a prospective analysis (ie, whether mood on one day influenced change in antibody production from that day to the next). In the second study, Stone et al. (35) used a similar design but a different sample population (96 members of an adult community instead of students). In this study subjects ingested the rabbit albumin pill on a daily basis for 12 consecutive weeks. They also completed a daily diary on each day. The diary included a daily life event checklist as well as positive and negative mood checklists. Finally, the investigators collected saliva samples on these same days for analysis of S-IgA antibody to rabbit albumin. Again, specific S-IgA levels were corrected for total S-IgA. As in the previous study, cross-sectional analyses indicated that subjects had lower specific S-IgA levels on days that they reported more negative moods and on days that they reported fewer positive moods. In addition, they had lower S-IgA levels on days they reported more negative events and on days they reported fewer positive events. In this case, however, having fewer positive events was more important than negative events. In this article the investigators also reported a prospective analysis, whether daily life events on one day predicted changes in antibody from that day to the next. In this case positive events on one day predicted higher antibody responses on the following 2 days.

The daily diary/challenge technique is a relatively unique approach. One difference between it and other paradigms discussed in this review is the close contiguity of stress and antibody measures. This allows examination of antibody production during or very soon after acute exposure to a stressor. This paradigm is also characterized by repeated daily measurements over multiple exposures. Hence the relations reported in these studies are associations that occur across multiple days of comparison. It is noteworthy that although daily diary studies allow for prospective lag analyses, the Stone et al. study primarily found crosssectional associations, leaving the possibility that immune changes triggered changes in reported affect and stress. This paradigm is well suited for studying stress- 
induced changes in S-IgA but could not be used to study changes in concentrations of IgG, which does not fluctuate on a daily basis.

Pneumococcal Immunization. There is a single study of the role of stress in children's response to pneumococcal immunization. As mentioned earlier, pneumococcal antigen is a polysaccharide and is processed differently than the antigens already discussed. Because polysaccharide antigens do not generate Bcell memory, all antibody responses to these antigens are considered primary. The pneumococcal vaccine consists of 23 separate polysaccharides, but antibody to only some of the antigens is usually determined.

Boyce et al. (36) studied primary immune response by immunizing 39 children (aged 4-6 years) with pneumococcal vaccine 1 week before the children started kindergarten. Antibody levels to three antibody classes (IgA, IgM, and IgG) for each of two antigens were assessed at immunization and 1 week after school started. Parents completed a questionnaire at the 1-week follow-up asking about behavioral problems their children manifested in response to starting school. They selected an arbitrary threshold for an antibody increase (determined by ELISA) as a positive response and counted the number of positive responses to six (3 antibody classes by 2 antigens) responses. Ratings of problem behavior were not associated with antibody response to the vaccine.

\section{DISCUSSION}

The evidence supports an association between psychological stress and humoral immune (antibody) response to immunization. This association is convincing in the case of secondary immune response but weak for primary response. There are actually very few studies on the role of stress in primary antibody response. These include two studies of response to the Hep B vaccine, one to KLH antigen, one to Rubella vaccine, and one to a pneumococcal vaccination. Two of the Hep B studies $(19,20)$, the KLH study (26), and the pneumococcal study (36) all failed to find relations between traditional negative measures of stress and primary response. Glaser et al. (20) found an association, but it was not with stress at the time of vaccination but rather to the average level of stress reported at the first, second, and third immunizations. Finally, in the rubella vaccine study (33), several measures of trait negative affect were associated with less primary response. Because this study was unlike the others in several ways, it is difficult to identify the critical difference that might have accounted for these discrepant results. Differences include the assessment of trait measures of stress (these imply that levels of negative affect were consistently high over the course of the study), the use of an attenuated viable virus, and the sample population (adolescent girls).

Overall the literature does not provide clear support for the hypothesis that psychological stress alters antibody response to a primary antigenic challenge. There are, however, a number of limitations of this work. One problem is that the studies varied widely in terms of the sensitivity of antibody assays and cutoffs chosen to indicate seropositivity. Another is that the literature is primarily based on response to or measurement of acute events or affective states, whereas the most convincing evidence of the effects of psychological stress on secondary response (see below) comes from those experiencing severe and chronic stressful events. Interestingly the only study finding an association between stress at the point of immunization and primary response used measures of enduring affective tone (33).

This lack of consistent associations between stress and suppression of primary antibody response in this literature contrasts with the results of animal research (see Ref. 37). Notable examples include the suppressive effects of social aggression (38), handling (39), and uncontrollable tail shock (40) on primary response to $\mathrm{KLH}$; and rotation stress (41), restraint stress (42), and social aggression $(43,44)$ on antibody response to inoculation with sheep red blood cells. These experimental studies provide a strong impetus to further examine the association between stress and primary response in humans.

In contrast to the relative lack of evidence of a primary response, promising evidence of an association between stress and secondary response has been found. The results of correlational studies of Hep B immunization are mixed in this regard. However, an impressive experimental study provided provocative evidence of an association (19). Those randomly assigned to an intervention (administered before the first immunization) designed to reduce chronic background stress had a greater increase in Hep B antibody concentration after the second and third immunizations. Because this intervention was targeted at relieving enduring sources of internal conflict, one might assume that the reduction in stress that occurs may be prolonged (even permanent) in nature. Consequently, those in the control (nontreatment) group would have elevated levels of stress over the entire course of the antibody production process, which would maximize the chance of influencing antibody production.

Unlike the Hep B literature, the correlational studies of influenza immunization show a very consistent pattern of results. Overall the four studies in this literature suggest that older adults confronted with an en- 
during and severe stressor (caring for a chronically ill spouse) develop antibody to influenza immunizations at a slower rate (and possibly less antibody overall) than nonstressed control subjects. There is evidence in these studies that the association between stress and suppressed antibody production was largest in response to viral antigens that had not been used in previous years' trivalent vaccines (29; J. KiecoltGlaser, personal communication, 2000). This is likely because of greater variability in antibody response to a new viral antigen. From a practical perspective, the emphasis in the influenza studies on older adults is laudable; however, it would be interesting to know whether other at-risk populations, such as people with chronic upper respiratory problems, and populations not at risk, such as healthy college students, show similar relations between stress and antibody production.

There was a failure to find an association between several measures of trait negative affect and secondary response to a rubella vaccination (33). These data are difficult to integrate here, outside the unique aspects of this challenge and the subject population described above.

Finally, oral antigen studies provide a somewhat different perspective on the relation of stress and secondary response. The daily assessment paradigm provides a strong temporal tie between acute stress levels and antibody levels. Consequently, we know from these data that at least in the case of S-IgA antibody response to oral antigen, stress on the same day as antigen challenge is associated with a decreased rate of antibody secretion on that day. Moreover, lower levels of positive affect were not only cross-sectionally associated with lower levels of antibody on the same day but also prospectively (controlling for same day antibody) associated with lower levels on the following 2 days. Because of the within-subject design used in these studies (stress days are compared with nonstressed days in the same subjects), we also know that individual differences that might play a role in assignment of subjects into stressed or nonstressed conditions in the other correlational studies are not an issue here.

In summary, the secondary antibody response literature is not consensual in its support of stress effects. However, there is sufficient support to conclude that stress is associated with less response under certain conditions. The conditions suggested by the literature include age (being 50 years old or older) and, most importantly, having a measure of stress that is relatively chronic or enduring. It is possible that the specific antigen challenge is also important (effects found for influenza vaccine, Hep B vaccine, and rabbit albu- min given orally), but the evidence is insufficient to suggest differences between antigens at this time. Clearly further work explicitly testing the role of these factors would be welcome.

\section{Other Predictive Variables}

There are suggestions across antigen challenges that measures other than the traditional negative stress assessments, such as life events, perceived stress, and negative affect, may be useful to pursue in the future. For example, three studies $(26,34,35)$ show an association with less positive affect and less production of antibody in response to a secondary challenge. Similarly (but not discussed in our review), less social support has been associated with less response to the second Hep B immunization (composite measure of antibody and blastogenic response) (20). Finally, another study suggested that individual differences in immune reactivity, suppression of mitogen-stimulated lymphocyte proliferation in response to an acute stress laboratory challenge, is associated with secondary antibody response (21).

\section{Clinical Importance}

In general, increases in levels of specific serum antibody to a viral immunization are associated with a decreased incidence of clinical illness when exposed to the virus (eg, 30-32). Hence the production of less antibody under stress would be associated with less protection. However, there are several limitations to existing work that raise questions about how important this is for clinical outcomes. First, the longest follow-up period in an immunization study is only 3 months after the final immunization. Hence it is possible that the effects of stress are on rate of antibody production but not on the final level of protection (45). Certainly longer follow-up periods would add considerably to this literature. Second, whether postimmunization antibody levels reach levels that provide greater protection is likely associated with antibody levels before immunization. Hence baseline levels must be taken into account when evaluating the effectiveness of an immunization and when evaluating stress effects on protective response to an immunization. Finally, the protection provided by an immunization is not attributable entirely to antibody production. For example, in the elderly, protection against influenza is thought to depend to some extent on cellular immune memory (T-cell response) as well (46). Consequently a broader view of immune response to immunization may be required in making inferences about the role 
stress in providing protection against infectious illness.

\section{Conceptual/Methodological Issues}

Probably the biggest source of variance across the studies in this literature has to do with timing of both the psychological and antibody measures. If we assume that stress levels need to be elevated at some (unknown) specific points during the primary or secondary production of antibody, then stress would need to be assessed at those appropriate points. However, this would not be an issue when studying either ongoing (chronic) stressful events or traitlike stress characteristics. Because those with enduring exposure or greater levels of trait (stable, dispositional) negative affective tone would have elevated stress levels over the entire course of response to the antigen, stress would be elevated at all points in the immune process. In contrast, acute measures of stress assess stress levels only during specific (near the time of the measure) points in the process. In fact, the literature supports the efficacy of using measures of enduring exposure or trait affect. Studies of a severe enduring stressor (caretaking for a sick spouse) consistently find that caretakers produce less antibody than control subjects. Similar consistency is found in studies using a trait measure of stress. This includes findings from two studies that trait negative affect predicted antibody response $(21,33)$ and that the intervention aimed at relieving enduring internal conflicts was also related to antibody response (19). Also included here is a study finding that immune stress-reactivity (21), thought to be an enduring trait as well (34), was associated with antibody production.

This emphasis on whether psychological stress occurs at the appropriate point in the process of antibody production suggests that "statelike" measures of stress will be most effective if they are closely tied to a "stress-critical" point in the antibody response process. For example, the studies where both stress and S-IgA antibody measures were done on a daily basis (and antibody changed on a daily basis) showed consistent associations between stress and antibody response, whereas studies in which state measures were given days, weeks, or months away from the outcome assessment produced extremely variable results. To move this literature forward, investigators need to consider the course of antibody response and generate hypotheses about the duration and timing of stressors that would influence that process (17).

Similar issues apply to when the follow-up measures of antibody level are collected. For example, although Jabaaij et al. (22) found an association be- tween stress and antibody levels 1 month after the third immunization, Petry et al. (23) failed to find a difference 3 months after immunization. It certainly is possible that a difference occurring 1 month after the immunization was no longer evident at 3 months. In summary, we need to more directly address questions of timing. For example, when in relation to immunization does the critical point at which stress influences antibody response occur? How long does it take for stress to significantly influence antibody production? How long do stress-induced changes last?

Statistical power is another issue that warrants more careful consideration in this literature. Assuming that existing studies provide accurate estimates of effect size, an influenza trial would need approximately 120 subjects (60 per group) to have sufficient statistical power (0.80) to detect postimmunization differences in antibody response between stressed and nonstressed groups. A Hep B trial would need approximately 140 subjects (70 per group) to achieve the same power. By these standards, few of the studies we reviewed (with the exception of Refs. 28 and 29) have a sufficiently large sample.

A final methodological issue has to do with the range of antibody responses to specific immunizations. Immunization protocols are carefully designed to induce a substantial immune response. Very successful immunizations may therefore leave relatively little room for individual differences. Consequently it is probable that stress and other psychosocial variables would play the greatest role in immunizations that are most variable in terms of raising antibody levels across people but would be less important in very effective immunizations. Consistent with this point is the difficulty one might expect in finding stress effects on repeated immunizations (such as influenza).

\section{Mediation}

Although there is evidence of a relation between psychological stress and secondary antibody production, the mechanisms responsible for this relation are not yet understood. As discussed earlier, stress might influence the immune processes involved in antibody production through its effects on behaviors that modulate immunity or through its effects on hormones that modulate the immune system. Variables that represent various pathways linking stress to antibody production have been measured in only a few studies. Glaser, Kiecolt-Glaser, and colleagues assessed the possible contribution of a range of potentially important behaviors, such as alcohol consumption, smoking status, nutrition, sleep, exercise, and weight gain, in all of their studies $(20,27,28)$, and similar measures were 
assessed by Marsland et al. (21). However, stressed and control populations did not differ on these behavioral measures in any of these studies. Consequently the assessed behaviors cannot be responsible pathways.

Two studies assessed the potential role of stresselicited elevations in cortisol. Vedhara et al. (29) found that cortisol levels of caregivers (based on three assessments before immunization) were greater than those of control subjects. A correlation between this basal cortisol measure and the antibody level 4 weeks after the influenza immunization suggests the possibility that cortisol partially mediated the maximal response. However, there was no direct test of mediation in the study (see Refs. 47 and 48). In their study of children's response to pneumococcal immunization, Boyce et al. (36) found that children with the greatest increases in cortisol in response to starting kindergarten had positive responses to fewer of the vaccine antigens. However, as mentioned earlier, their measure of psychological stress (behavioral problems) was not associated with antibody production. Finally, although the studies of S-IgA response to daily stress did not assess cortisol, there is evidence that stressful daily events are associated with same-day rises in cortisol (49). Again, this is consistent with but not direct evidence of the hypothesis that cortisol mediates the association between daily stress and daily antibody response (see Ref. 50). Overall the studies reviewed here suggest the potential importance of cortisol as a pathway through which psychological stress alters antibody production in response to a vaccine. It is noteworthy, however, that there are a range of other hormones that might play a role in mediating the association between stress and secondary antibody response, including epinephrine and norepinephrine, prolactin, and growth hormone $(7,51)$. Clearly further work is necessary to clarify the biological changes that accompany stress and influence antibody response.

\section{CONCLUSIONS}

In summary, the evidence we reviewed suggests a relationship between psychological stress and secondary immune response. Moreover, these associations may be amplified among the elderly and possibly other populations with vulnerable immune systems. Unfortunately there are only hints as to possible pathways linking stress and antibody production. In contrast, the few studies examining primary response provide little evidence of an effect. Future work that pays greater attention to the temporal course of stress (eg, acute or chronic) in relation to the kinetics of the antibody response would help provide a more definitive conclusion. Moreover, studies with longer follow-up pe- riods would provide evidence on whether associations with stress involve only the rate of antibody production or involve the highest level of protection reached in response to the immunization.

We thank the John D. and Catherine T. MacArthur Foundation Network on Socioeconomic Status and Health, which stimulated us to write this review. Collaboration of the authors was facilitated by the Pittsburgh Mind-Body Center (Grants HL65111 and HL65112). Gregory Miller's participation was supported by a postdoctoral fellowship from the National Institute of Mental Health (Grant MH18269), and Sheldon Cohen's participation was supported by a Senior Scientist Award from the National Institute of Mental Health (MH00721). We appreciate the input provided by John Sheridan and the blind reviewers.

\section{REFERENCES}

1. Cohen S, Herbert TB. Health psychology: psychological factors and physical disease from the perspective of human psychoneuroimmunology. Annu Rev Psychol 1996;47:113-42.

2. Herbert TB, Cohen S. Stress and immunity in humans: a metaanalytic review. Psychosom Med 1993;55:364-79.

3. Kiecolt-Glaser JK. Norman Cousins Memorial Lecture 1998 Stress, personal relationships, and immune function: health. Brain Behav Immun 1999;13:61-72.

4. Kemeny ME, Gruenewald TL. Psychoneuroimmunology update. Semin Gastrointest Dis 1999;10:20-9.

5. Stone AA, Mezzacappa ES, Donatone BA, Gonder M. Psychosocial stress and social support are associated with prostatespecific antigen levels in men: results from a community screening program. Health Psychol 1999;18:482-6.

6. Benjamini E, Leskowitz S, Sunshine G. Immunology: a short course. 3rd ed. New York: Wiley-Liss; 1996.

7. Rabin BS. Stress, immune function, and health: the connection. New York: Wiley-Liss; 1999.

8. Kraehenbuhl JP, Neutra MR. Molecular and cellular basis of immune protection of mucosal surfaces. Physiol Rev 1992;72: 853-79.

9. Lamm ME. Interaction of antigens and antibodies at mucosal surfaces. Annu Rev Microbiol 1997;51:311-40.

10. Tomasi TB. The discovery of secretory IgA and the mucosal immune system. Immunol Today 1992;13:416-8.

11. Weiner HL, Friedman A, Miller A, Khoury SJ, Al-Sabbagh A, Santos L, Sayegh M, Nussenblatt RB, Trentham DE, Hafler DA. Oral tolerance: immunologic mechanisms and treatment of animal and human organ-specific autoimmune diseases by oral administration of autoantigens. Annu Rev Immunol 1994;12:809-37.

12. Ader R, Felten DL, Cohen N, editors. Psychoneuroimmunology. 3rd ed. New York: Academic Press; 2001.

13. Glaser R, Kiecolt-Glaser J. editors. Handbook of human stress and immunity. San Diego (CA): Academic Press; 1994.

14. Kiecolt-Glaser JK, Glaser R. Methodological issues in behavioral immunology research with humans. Brain Behav Immun 1988; 2:67-78.

15. Schwartz R, Dameshek W. Drug-induced immunologic tolerance. Nature 1959;183:1682-3.

16. Cohen S, Evans GW, Stokols D, Krantz DS. Behavior, health, and environmental stress. New York: Plenum Press; 1986. 
17. Cohen S, Kessler RC, Gordon L. Strategies for measuring stress in studies of psychiatric and physical disorder. In: Cohen S, Kessler RC, Underwood Gordon L, editors. Measuring stress: a guide for health and social scientists. New York: Oxford Press; 1995. p. 3-26.

18. Francis DP, Hadler SC, Thompson SE, Maynard JE, Ostrow DG, Altman N, Braff EH, O’Malley P, Hawkins D, Judson FN, Penley K, Nylund T, Christie G, Meyers F, Moore JN Jr, Gardner A, Dolo IL, Miller JH, Reynolds GH, Murphy BL, Schable CA, Clark BT, Curran JW, Redeker AG. The prevention of hepatitis B with vaccine. Ann Intern Med 1982;97:362-6.

19. Petrie KJ, Booth RJ, Pennebaker JW, Davison KP, Thomas MG. Disclosure of trauma and immune response to a hepatitis B vaccination program. J Consult Clin Psychol 1995;63:787-92.

20. Glaser R, Kiecolt-Glaser JK, Bonneau RH, Malarkey WB, Kennedy S, Hughes J. Stress-induced modulation of the immune response to recombinant hepatitis B vaccine. Psychosom Med 1992;54:22-9.

21. Marsland AL, Cohen S, Rabin BS, Manuck SB. Associations between stress, trait negative affect, acute immune reactivity, and antibody response to hepatitis B vaccination. Health Psychol. In press.

22. Jabaaij L, Grosheide PM, Heijtink RA, Duivenvoorden HJ, Ballieux RE, Vingerhoets AJJM. Influence of perceived psychological stress and distress on antibody response to low dose rDNA hepatitis B vaccine. J Psychosom Res 1993;37:361-9.

23. Petry LJ, Weems LB, Livingstone JN. Relationship of stress, distress, and the immunologic response to a recombinant hepatitis B vaccine. J Fam Pract 1991;32:481-6.

24. Jabaaij L, van Hattum J, Vingerhoets AJJM, Oostveen FG, Duivenvoorden HJ, Ballieux R. Modulation of immune response to rDNA hepatitis B vaccination by psychological stress. J Psychosom Res 1996;41:129-37.

25. Pennebaker JW. Writing about emotional experiences as a therapeutic process. Psychol Sci 1997;8:162-6.

26. Snyder BK, Roghmann KJ, Sigal LH. Effect of stress and other biopsychosocial factors on primary antibody response. J Adolesc Health Care 1990;11:472-9.

27. Kiecolt-Glaser JK, Glaser R, Gravenstein S, Malarkey WB, Sheridan JF. Chronic stress alters the immune response to influenza virus vaccine in older adults. Proc Natl Acad Sci USA 1996;93:3043-7.

28. Glaser R, Kiecolt-Glaser JK, Malarkey WB, Sheridan JF. The influence on psychological stress on the immune response to vaccines. Ann NY Acad Sci 1998;840:649-655.

29. Vedhara K, Cox NKM, Wilcock GK, Perks P, Hunt M, Anderson S, Lightman SL, Shanks NM. Chronic stress in elderly caregivers of demential patients and antibody response to influenza vaccination. Lancet 1999;353:627-31.

30. Belshe RB, Gruber WC, Mendelman PM, Mehta HB, Mahmood K, Reisinger K, Treanor J, Zangwill K, Hayden FG, Bernstein DI, Kotloff K, King J, Piedra PA, Block SL, Yan L, Wolff M. Correlates of immune protection induced by live, attenuated, coldadapted, trivalent, intranasal influenza virus vaccine. J Infect Dis 2000;181:1133-7.

31. Clements ML, Betts RF, Tierney EL, Murphy BR. Serum and nasal wash antibodies associated with resistance to experimental challenge with influenza A wild-type virus. J Clin Microbiol 1986;24:157-60.

32. Gravenstein S, Drinka P, Duthie EH, Miller BA, Brown S, Hensley M, Circo R, Langer E, Ershler WB. Efficacy of an influenza hemagglutinin-diptheria toxoid conjugate vaccine in elderly nursing home subjects during an influenza outbreak. J Am Geriatr Soc 1994;42:245-51.

33. Morag M, Morag A, Reichenberg A, Lerer B, Yirmira R. Psychological variables as predictors of rubella virus antibody titers and fatigue: a prospective, double-blind study. J Psychiatr Res 1999;5:389-95.

34. Stone AA, Cox DS, Valdimarsdottir H, Jandorf L, Neale JM. Evidence that secretory IgA antibody is associated with daily mood. J Pers Soc Psychol 1987;52:988-93.

35. Stone AA, Neale JM, Cox DS, Napoli A, Valdimarsdottir H, Kennedy-Moore E. Daily events are associated with a secretory immune response to an oral antigen in men. Health Psychol 1994;13:440-6.

36. Boyce WT, Adams S, Tschann JM, Cohen F, Wara D, Gunnar MR. Adrenocortical and behavioral predictors of immune responses to starting school. Pediatr Res 1995;38:1009-17.

37. Moynihan JA, Cohen N, Ader R. Stress and immunity. In: Scharrer B, Smith EM, Stefano GB, editors. Neuropeptides and immunoregulation. New York: Springer-Verlag; 1994. p. 120-38.

38. Fleshner M, Laudenslager ML, Simons L, Maier SF. Reduced serum antibodies associated with social defeat in rats. Physiol Behav 1989;45:1183-7.

39. Moynihan JA, Koota D, Brenner G, Cohen N, Ader R. Repeated intraperitoneal injections of saline attenuate the antibody response to a subsequent intraperitoneal injection of antigen. Brain Behav Immun 1989;3:90-6.

40. Laudenslager M, Fleshner M, Hofstadter P, Held PE, Simons L, Maier F. Suppression of specific antibody production by inescapable shock: stability under varying conditions. Brain Behav Immun 1988;2:92-101.

41. Esterling B, Rabin BS. Stress-induced alteration of T-lymphocyte subsets and humoral immunity in mice. Behav Neurosci 1987;101:115-9.

42. Okimura T, Nigo Y. Stress and immune responses. I. Suppression of T-cell functions in restraint-stressed mice. Jpn J Pharmacol 1986;40:505-11.

43. Beden SN, Brain PF. Studies on the effect of social stress on measures of disease resistance in laboratory mice. Aggress Behav 1982;8:126-9.

44. Beden SN, Brain PF. Effects of attack-related stress on the primary immune response to sheep red blood cells in castrated mice. IRCS Med Sci 1984;12:675.

45. Cunnick JE, Cohen S, Rabin BS, Carpenter B, Manuck S, Kaplan J. Alterations in specific antibody production due to rank and social instability. Brain Behav Immun 1991;5:357-69.

46. McElhaney JE, Meneilly GS, Lechelt KE, Bleackley RC. Splitvirus influenza vaccines: do they provide adequate immunity in the elderly? J Gerontol Med Sci 1994;49:37-43.

47. Baron RA, Kenny DA. The moderator-mediator variable distinction in social psychological research: conceptual, strategic, and statistical consideration. J Pers Soc Psychol 1986;51:1173-82.

48. Stone AA. Selected methodological concepts: mediation and moderation, individual differences, aggregation strategies, and variability of replicates. In: Schneiderman N, McCabe P, Baum A, editors. Stress and disease processes: perspectives in behavioral medicine. Hillsdale (NJ): Lawrence Erlbaum Associates; 1992. p. 55-71.

49. Van Eck M, Berkhof H, Nicolson N, Sulon J. The effects of perceived stress, traits, mood states, and stressful daily events on salivary cortisol. Psychosom Med 1996;58:447-58.

50. Coe CL, Rosenberg LT, Fisher M, Levine S. Psychological factors capable of preventing the inhibition of antibody responses in separated infant monkeys. Child Dev 1987;58:1420-30.

51. Wu H, Wang J, Cacioppo JT, Glaser R, Kiecolt-Glaser JK, Malarkey WB. Chronic stress associated with spousal caregiving of patients with Alzheimer's dementia is associated with downregulation of B-lymphocyte GH mRNA. J Gerontol 1999;54A:M212-5. 